\title{
Verband katholischer Tageseinrichtungen für Kinder (KTK) - Bundesverband e.V. (Hg.) (2020). Vielfältige Teams. Die Religionszugehörigkeit von Fachkräften in katholischen Kitas. Freiburg: Verband katholischer Tageseinrichtungen für Kinder. ISBN 978-3-933383-45-7. 258 Seiten.
}

\section{Agnes Wuckelt}

Katholische Hochschule Nordrhein-Westfalen (a.wuckelt@katho-nrw.de)

Der erste Band der Reihe „Religionspädagogik der frühen Kindheit“, herausgegeben vom KTK-Bundesverband, dokumentiert dessen Religionspädagogische Jahrestagung 2019 zum Thema "Auftrag und Selbstverständnis religiös pluraler Teams in katholischen Kindertageseinrichtungen.“ Dazu gesellen sich themenbezogene Beiträge, die die Fragestellungen der Tagung aufgreifen und erweitern. Teil I (21-103) beschreibt Dimensionen von Vielfalt, die in Kitas als Problemlagen auftreten: die diözesane Verschiedenheit des katholischen Arbeitsrechts (Röser) sowie die Unterschiede in der Kirchenmitgliedschaft von Mitarbeitenden in kirchlichen Organisationen (Ebertz) und die sich daraus ergebenden Fragen für die Anstellung und Arbeit religiös gemischter Teams in katholischen Kitas (Wollasch). Teil II (107-203) zeigt Lösungsansätze auf: in Form einer Reform des kirchlichen Arbeitsrechts (Beer \& Colloseus), im glaubwürdigen Führungsverhalten kirchlicher Träger, die die Frage der Kirchlichkeit nicht nur auf die Mitarbeitenden abwälzen (Cremer), im Konzept einer pluralitätsfähigen Religionspädagogik, die sowohl der religiösen Vielfalt bei den Kindern also auch im Team Rechnung trägt (Hugoth), in einem modularisierten Prozessmodell, nach dem sich ein Team über den Erwerb von Grundwissen zu den Weltreligionen bis zur Erfahrung und Gestaltung der Kita als Ort gelebter Begegnung mit Gott in interreligiöse Arbeit einübt (Wollasch), im Akzeptieren einer gestuften Loyalität in religiös pluralen Teams (Bündgens) sowie im Bemühen, der Vielfalt bei den Kindern und ihren Familien wie der religiösen Vielfalt im Team gerecht zu werden (Jansen). Teil III (207-254) bietet Praxisberichte: aus dem bundesweit ersten christlich-muslimischen Kindergarten "Abrahams Kinder“ in Gifhorn (Wrasmann \& Minkus), aus der pastoralen Begleitung von Kita-Teams (Hardy \& Husen), aus dem Integrationskindergarten Regenbogen der Caritas München (Horlbeck). Den Abschluss des Bandes bildet die Auswertung der Arbeitsgruppen der Jahrestagung.

Geboten wird ein bunter Strauß von Aspekten und Perspektiven, bestimmt vom mehrfachen Plädoyer für Vielfalt in katholischen Kindertageseinrichtungen. Unumstritten ist die Notwendigkeit des Wahrund Ernstnehmens der gesellschaftlichen Pluralität im Rahmen des pastoral-diakonischen Auftrags der Kirche in einer pluralen Gesellschaft mit ständig zunehmender Vielfalt (Ebertz). Nicht nur Kinder und Familien mit Anbindung an eine Gemeinde, sondern auch Kinder und ihre Familien, „die sich im sakramentalen oder kirchenrechtlichen Sinn nicht als Kirchenmitglieder sehen“, sind willkommen und „ein gleichgewichtiger und gleichwertiger Teil“ der Gemeinschaft der Kita (Ebertz: 15; dazu auch Wollasch: 79-83). Zum Problem kann jedoch werden, dass auch innerhalb der Gruppe der pädagogischen Fachkräfte religiöse Vielfalt gegeben ist: „Menschen mit unterschiedlichem religiösem Bewusstsein, unterschiedlicher religiöser Überzeugung, Lebenseinstellung und Alltagspraxis“ (Hugoth: 125; dazu auch Ebertz; Bündgens). So erweist sich die „Personalfrage“ insofern als „Testfall“, als das Akzeptieren von Vielfalt in den Teams „noch längst nicht in vergleichbarem Maß angekommen“ ist (Wollasch: 84).

In diesem Kontext stellt sich die Frage nach dem kirchlichen Arbeitsrecht, konkret der „Grundordnung des kirchlichen Dienstes“ (Röser). Die Umsetzung dieser Rahmenordnung in die Praxis unterscheidet sich von Diözese zu Diözese und führt zu einer unterschiedlichen Einstellungspraxis (Röser: 28). Zudem 
werden auch an das „außerdienstliche Verhalten“ der Mitarbeitenden besondere Anforderungen gestellt, sog. „Loyalitätsobliegenheiten“, die „arbeitsrechtlich die Glaubwürdigkeit des kirchlichen Dienstes sichern“ sollen (Röser: 34). Auf diesem Hintergrund ist zu fragen, ob Einrichtungen „nur dann ihren Dienst im Sinne ihres kirchlichen Auftrags erfüllen“, wenn zumindest die Mehrheit ihrer Mitarbeitenden diesen Anforderungen entspricht (Cremer: 120). Dem wird entgegengehalten, dass „die Identität einer kirchlichen Organisation [...] viele Menschen unterschiedlicher Biographien, Prägungen und Glaubenshaltungen integrieren und dabei einen glaubwürdigen Dienst am Menschen leisten" kann (Cremer: 121) - und zwar dann, wenn der christliche Auftrag „im Führungsverhalten der Verantwortlichen" gelebt wird, die "das Problem der Kirchlichkeit nicht einfach bei ihren Mitarbeitenden“ abladen oder „über deren vermeintlich oder real fehlende kirchliche Sozialisation“ klagen (Cremer: 122).

Erforderlich ist daher eine Reform des kirchlichen Arbeitsrechts die anerkennt, dass „auch kirchliche Mitarbeitende ihr Leben auf sehr vielfältige Weise leben“ und fragt, „wie der kirchliche Sendungsauftrag in Anerkennung dieser Vielfalt erfüllt werden kann“ (Beer \& Colloseus: 111). Dazu „brauchen wir auch Teams, die Zeichen für die gelingende Integration, für die Fähigkeit zur Pluralität sind“ (Beer \& Colloseus: 113). Rechtliche Regelungen dürfen nicht an die Stelle des Sendungsauftrags der Kirche gesetzt werden. „Wo alle das ihre zur Glaubwürdigkeit beitragen, können wir hoffentlich zeigen, worum es uns geht: Nicht um Sanktionen, sondern um eine Vision“ (Beer \& Colloseus:113).

Religionspädagogisch betrachtet brauchen katholische Kindertageseinrichtungen Profilierungen, die den Sendungsauftrag katholischer Kitas neu definieren: „,als Lernort des Glaubens für Kinder und Erwachsene" (Hugoth: 129). Nötig sind "neue theologische und religionspädagogische Denkfiguren", wie die Denkfigur der „Religionsproduktion“ - auch im Blick auf die pädagogischen Fachkräfte. Auch „nicht konfessionsgebundene“ Erwachsene beteiligen sich „, an religionsproduktiven Prozessen mit Kindern und im Team" (Hugoth: 138).

Es geht „um das Teilen von Lernerfahrungen als gemeinsame Schritte auf einem Weg“, einen "religionssensiblen Zugang“, der es auch einer muslimischen oder nicht-christlichen Kollegin ermöglicht, Teil des Teams zu werden (Wollasch: 144f.). Der Idee „Institutionenorientierung vor Personenorientierung“ folgend bedeuten - im engeren wie im weiteren Sinne - multireligiöse Teams „keine Infragestellung der Konfessionalität, wohl aber eine konzeptionelle Weiterentwicklung.“ Konfessionalität ist „vorrangig an der Institution und der Verantwortung des Trägers" festzumachen (Bündgens: 177). Darüber hinaus gilt: „Pluralität gefährdet die Glaubwürdigkeit nicht, im Gegenteil: Wenn Pluralität gelingt, ist das ein starkes Zeichen von Glaubwürdigkeit. Loyalität ist nicht der Versuch, Uniformität zu erzwingen“ (Bündgens: 186; dazu auch Beer \& Colloseus: 111; Jansen: 201). So führt die „Frage nach religiös pluralen Teams in katholischen Kitas [...] zu Grundthemen des Glaubens und bleibt eine lohnende Herausforderung für Theologie und Praxis“ (Bündgens: 190).

Band 1 von „Religionspädagogik der frühen Kindheit“ des KTK thematisiert mit der Diskussion einer gegebenen wie einzufordernden Vielfalt von Kita-Teams ein Dauerthema kirchlicher Einrichtungen. Wieviel Verbundenheit und Identifikation mit der Kirche als sakramentaler Gemeinschaft sollen katholische Mitarbeitende einbringen? Führt der Einsatz von nicht-katholischen Fachkräften zur Aufgabe des christlichen Sendungsauftrags, Mensch und Welt im Licht des Evangeliums zu deuten? Die Frage nach der "Glaubwürdigkeit" kirchlicher Einrichtungen und Kirche durchzieht die Beiträge des Bandes. Sie ist die Kernfrage, um die es sich dreht, wo Kirche nach innen und in die Öffentlichkeit wirkt. Eine Antwort: Es braucht Vielfalt, das gleichberechtigte Zusammenwirken aller, um das Evangelium im Hier und Heute glaubwürdig verkünden zu können. Dazu bietet der Band vielfältige Anregungen zur weiteren Diskussion. 